\title{
Assessment of Respondent Driven Sampling for Recruiting Female Sex Workers in Two Vietnamese Cities: Reaching the Unseen Sex Worker
}

\author{
Lisa Grazina Johnston, Keith Sabin, \\ Mai Thu Hien, and Pham Thi Huong
}

\begin{abstract}
Respondent driven sampling (RDS) is a relatively new method to sample hard-to-reach populations. Until this study, female sex workers (FSWs) in Vietnam were sampled using a variety of methods, including time location sampling (TLS), which may not access the more hidden types of FSWs. This paper presents an analysis from an HIV biological and behavioral surveillance survey to assess the feasibility and effectiveness of RDS to sample FSWs, to determine if $R D S$ can reach otherwise inaccessible FSWs in Vietnam and to compare RDS findings of HIV risk factors with a theoretical TLS. Through face-to-face interviews with FSWs in Ho Chi Minh City (HCMC) and Hai Phong (HP), data were collected about the venues where they most often solicit their clients. These data were used to create three variables to assess whether FSWs solicit their clients in locations that are visible, semi-visible and nonvisible. For this analysis, the visible group simulates a sample captured using TLS. Survey results in HIV prevalence and related risk factors and service utilization, adjusted for sampling methodology, were compared across each of the three FSW visibility groups to assess potential bias in TLS relative to RDS. The number of selfreported visible FSWs (HCMC: $n=311$; HP: $n=162$ ) was much larger than those of the semi-visible (HCMC: $n=65$; HP: $n=43$ ) and non-visible (HCMC: $n=37$; HP: $n=$ 10) FSWs in HCMC and HP. Non-visible FSWs in both cities were just as likely as visible and semi-visible FSWs to be HIV positive (HCMC: visible 14.5\%, semi-visible $13.8 \%$, non-visible $13.5 \%$, p value $=0.982$; HP: visible $35.2 \%$, semi-visible $30.2 \%$, non-visible $30.0 \%$, p value $=0.801)$, to practice behaviors that put them at risk for contracting and transmitting HIV (injecting drug use-HCMC: visible $13.8 \%$, semivisible $12.3 \%$, non-visible $5.4 \%$, p value $=0.347$; HP: visible $38.9 \%$, semi-visible $23.3 \%$, non-visible $30.0 \%$, p value $=0.378$, to have no condom use in the past month -HCMC only: visible $52.7 \%$, semi-visible $63.1 \%$, non-visible $48.6 \%$, p value = 0.249 ) and to have symptoms of a sexually transmitted infection (STI) in the past year (HCMC: visible $16.1 \%$, semi-visible $12.3 \%$, non-visible $16.2 \%$, p value $=0.742 ; \mathrm{HP}$ : visible $13.6 \%$, semi-visible $18.6 \%$, non-visible $20.0 \%$, p value $=0.640$ ). There was a difference found among the visible, semi-visible and non-visible groups in HP for no past month condom use (visible $53.1 \%$, semi-visible $79.1 \%$, non-visible $60.0 \%$, $p$ value $=0.009)$. This study found that RDS was successful at recruiting hidden types of FSWs in Vietnam. Past reports of FSWs in Vietnam have assessed the more visible FSWs
\end{abstract}

\footnotetext{
Lisa Grazina Johnston (independent consultant), Johnston and Sabina are with the Centers for Disease Control and Prevention, Atlanta, USA; Hein is with the LIFE-GAP Office, Ministry of Health, Hanoi, Vietnam; Houng is with the Centers for Disease Control and Prevention, Hanoi, Vietnam; Johnston is an independent consultant.

Correspondence: Lisa Grazina Johnston, 29 Camino Botanica, Santa Fe, NM 87607, USA. (E-mail: lsjohnston@comcast.net)
} 
as being the most vulnerable and at risk for HIV. Although the number of visible FSWs is much higher than those of the semi and non-visible groups, this study found that the nonvisible FSWs are very vulnerable to HIV infection. If prevention programs are targeting and responding to those who are most likely to be assessed (e.g., more visible types of FSWs) then this analysis indicates that a significant proportion of the FSW population at risk for HIV may not be receiving optimal HIV information and services.

KEYWORDS HIV, Respondent-driven sampling, Sex workers, Vietnam.

\section{INTRODUCTION AND BACKGROUND}

Vietnam is in the midst of an HIV epidemic concentrated among injection drug users (IDUs) and, increasingly, female sex workers (FSWs). In recent years, the epidemic in Vietnam appears to be moving rapidly from IDU populations to FSWs through sexual contact with injecting male clients and the adoption of injection drug use behaviors by FSWs. Sexual transmission of HIV from FSWs to their non-injecting clients, who in turn have non-commercial sex partners, greatly increases the probability that HIV will spread to the general population. In 2002, the Department of Social Evils in the Ministry of Labor, Invalids, and Social Affairs estimated that 130,000 women work as sex workers in Vietnam (other estimates put the number much higher). ${ }^{1-3}$

To understand and respond to HIV transmission patterns in Vietnam, numerous surveys have measured HIV prevalence and related risk factors among FSWs. However, the lack of sampling frames, relative rarity of this population, and their stigmatized and/or illegal behaviors, hinder investigators from obtaining representative samples of FSWs. Further, it is important to recognize the heterogeneity of the sex worker population. Broadly, FSW can be dichotomized into direct and indirect sex workers. Indirect sex workers typically have a primary job or source of income other than sex work, though that job may lead to contacts with potential clients (e.g., bar maids or masseuses). Direct sex workers typically do not have another job. Direct sex workers can be further broken down into street-based, brothel-based, or gatekeeper-based. The latter may involve a formal "pimp"/agent relationship or informal referrals from hotel concierges or taxi drivers. Use of the Internet to find clients is increasing also.

There is a concerted effort by the Government of Vietnam to reduce prostitution by placing FSWs in administrative detention in government rehabilitation centers where they can spend anywhere from 1 month to several years. Fear of detention leads FSWs to exercise greater caution and more subtlety on the streets. Recently, FSWs have become even more difficult to reach because the Vietnamese government is imposing penalties (fines, jail sentences and possible closure) on the owners of entertainment establishments where sex is allegedly sold. ${ }^{4}$ As a result, FSWs will now wait off-site for an establishment owner or some other gatekeeper to contact them at a client's request.

FSWs are also hard to reach because they are highly mobile and move regularly from bar to bar, between districts and towns, and within and to other provinces. ${ }^{5-9}$ Some FSWs are difficult to locate because they have primary jobs and engage in sex work part-time. For instance, women from rural areas may travel to urban areas to sell agricultural goods and only sell sex as a way to earn extra income. ${ }^{5}$ Higher paid FSWs, such as those who solicit clients from scooters, the Internet, through agents and over the telephone, have the financial capacity to remain hidden. 5,10 
Past efforts to survey FSWs in Vietnam, including annual sentinel HIV surveillance, have relied on convenience samples from rehabilitation centers and street-based FSWs. These samples comprise the type of FSWs who are reportedly the most vulnerable, and therefore most at risk, and visible, (i.e., visible enough to be captured by the police or found by public health staff) and do not represent the entire sex work community. ${ }^{5}$ Further efforts to assess HIV related risk factors among FSWs have used other non-probability sampling methods, such as institutional sampling, targeted and chain referral sampling. ${ }^{11-16}$ FSWs in Vietnam have also been sampled using a probability sampling method called time location sampling (TLS), also known as time-space or venue-day-time sampling. ${ }^{17,18}$ However, TLS can only be representative of those FSWs who are easily located at visible sites, thereby missing potentially important information from other types of FSWs.

The Vietnamese Ministry of Health, with technical assistance from Centers for Disease Control and Prevention-Global AIDS Program, conducted a survey using a relatively new sampling method to assess HIV prevalence, risk factors and service utilization among FSWs. The goal of the survey was to provide baseline information before increasing numbers of HIV prevention programs targeting these populations were initiated. This sampling method, known as respondent driven sampling (RDS), is a variant of chain-referral sampling that utilizes an incentive for being interviewed and another incentive for recruiting peers (other FSWs) to be interviewed. ${ }^{19-22}$ Sampling begins by selecting a small number of non-randomly selected members of the target population, known as seeds, to take part in the study. Seeds initiate the chain referral by recruiting a fixed number of peers who, in turn, recruit other peers. The process continues until the target sample size is attained. Links among peers are recorded using anonymous identification numbers.

Although RDS is relatively new to public health researchers, it is starting to be used widely in place of other well known types of sampling methods for hard to reach populations. Researchers are considering the advantages of using RDS in place of TLS, the only other probability method for hard to reach populations, to conduct future HIV biological and behavioral surveillance surveys. TLS has been used to sample FSWs who gather and are accessible at public locations (or venues) that can be listed in a sampling frame. ${ }^{23-25}$ TLS entails identifying days and times when the target population gathers at specific locations (e.g., brothels, city blocks, bars), constructing a sampling frame of time and location units, randomly selecting these units and systematically intercepting consenting members of the target population for interviews. ${ }^{24-26}$ When implemented correctly, TLS allows researchers to construct a sample with known probabilities, make statistical inferences to the larger population of venue visitors, and theorize about the introduction of biases that may limit generalization of results to the target population. ${ }^{23}$

Two TLS surveys of FSWs in Vietnam have been reported. In 2000 Family Health International (FHI) conducted a behavioral survey with karaoke $(n=2,302)$ and street-based $(n=1,300)$ FSWs in five Vietnamese cities using TLS. ${ }^{18}$ During 2003, the Vietnamese National Institute of Hygiene and Epidemiology collected data from karaoke $(n=1,367)$ and street-based $(n=976)$ FSWs from five provinces. ${ }^{17}$

Although TLS can produce a probability sample by ensuring that time-location units are enumerated and selected randomly, it allows researchers to reach only those segments of the population who are visible and identifiable. Depending on the population of interest, this may introduce a bias by differential sampling of the portion of the population which is most at-risk. TLS can be expected to miss those segments that are less visible (e.g., FSWs who solicit clients through agents, by 
telephone or Internet). The "visible" portion of a population does not represent the whole population any more than an institution or clinic-based population does; "hidden" population members have a zero probability of selection, reducing representativeness of the sample.

The objectives of this paper are to present findings from a survey on HIV prevalence, risk behaviors and service utilization to (1) assess the effectiveness of RDS to sample FSWs in Vietnam; and (2) to compare RDS findings with theoretical TLS. This is especially important given that evaluations of RDS used for HIV surveillance have only come from IDUs and males who have sex with males populations in the United States..$^{20,22,27-29}$ Until this study, RDS has not been evaluated in Asia among FSWs. This methodology warrants investigation to assess its ability to sample hard to reach populations in international settings.

\section{MATERIALS AND METHODS}

Data to evaluate RDS were collected from FSWs in Ho Chi Minh City (HCMC) and Hai Phong (HP), Vietnam. Eligible participants were over the age of 18 and had exchanged sex for money in the previous month. FSWs were recruited by their peers (other FSWs) and provided a small monetary incentive for completing a questionnaire and providing a saliva sample to test for HIV (US $1 \$ 3$ in HP and US I\$4 in HCMC). FSWs were provided another incentive for recruiting up to three other FSWs to participate (approximately US $\backslash \$ 1$ in HP and US $\backslash \$ 1.50$ in HCMC for each eligible recruit). Data were gathered from FSWs in HP from April to June and in HCMC from May to July, 2004. Recruitment began with 20 FSWs, nonrandomly selected by survey staff, in each city. Outreach workers selected these initial recruits, or "seeds," based on where they most often solicited their clients. A majority of the seeds were from the visible groups, street-based and karaoke-based FSWs. A few seeds from hotels and guesthouses were recruited.

Once seeds consented, they completed a one-hour face-to-face interview with a trained Vietnamese interviewer. The interview instrument consisted of questions about the participant's demographic background, current and past drug use, sexual behaviors, HIV knowledge, risk perceptions and service utilization, and about where they usually solicit their clients. After the interview, survey staff provided participants with three coupons with which to recruit up to three other FSWs who were coworkers, friends, acquaintances or family members. Each coupon contained a unique identification number and provided basic information about the study and the addresses of the interview sites. Respondent/recruiters were asked to return to collect their incentives for successful recruits. A brief follow-up questionnaire was administered during this visit to ascertain the recruiter's relationship to their recruits and the number and type of refusals they received. This permitted the assessment of participation bias due to refusals or no-shows.

Oraquick $^{\mathrm{TM}}$ HIV antibody rapid test kits for oral fluids were used for HIV testing. No one refused to provide an oral sample. Participants did not learn their test results at the interview site but were provided a voucher to receive a free HIV test with counseling at a nearby voluntary counseling and testing (VCT) center.

For the assessment of bias in a theoretical TLS-drawn sample in comparison to an actual RDS-drawn sample, respondents were classified as accessible, i.e., visible to TLS, if they reached their clients in a public venue. Categories were established from the response to the question, "Where do you usually reach your clients?" Responses were aggregated into three groups: visible (street, park, karaoke bar); 
semi-visible (brothel, guesthouse, hotel); non-visible (agent, telephone, Internet). Our assumption is that the visible group could potentially be captured by TLS. The semi visible group may or may not be captured with TLS, and the non-visible group would be missed with TLS."

\section{STATISTICAL ANALYSIS}

Adjusted proportions and 95\% confidence intervals (ci) for FSWs' level of visibility were analyzed using the RDS analysis tool (RDSAT). ${ }^{30}$ RDSAT adjusts proportion estimates for participants' network sizes and recruitment patterns. ${ }^{20,31}$ RDSAT adjustments use principles of social network theory to account for biases found in conventional chain referral sampling. ${ }^{31,32}$

Chi-square proportions and $p$-values for comparisons of HIV risk factors and service utilization of RDS and theoretical TLS were analyzed using SPSS 11.0. Five of the 20 non-randomly selected seeds in the HP sample, and four seeds in the HCMC sample were not used in the final analysis since they did not recruit any peers. It is not known why these seeds did not recruit anyone. The sample size was calculated based on $15 \%$ increase in use and knowledge of CDC sponsored VCT HIV services and outreach. We estimated low baseline knowledge and use of services (no actual data available) with high HIV prevalence. HP sample size was 200 based on higher knowledge from existing health education programs. The sample size in HCMC was 400, assuming lower baseline knowledge. Design effect was set at 2 .

This study was reviewed by the Associated Director for Science of the National Center for HIV, STD and TB prevention at CDC who classified this as a surveillance

\footnotetext{
"Definitions: Visible FSWs Street-based FSWs wait for clients to approach them and will usually provide sex in a hotel rooms or in some public location such as in an alley way or park. The streets and parks where FSWs congregate are often used in TLS since these types of FSWs are often accessible for interviewing and can be identified and enumerated by researchers. Although most entertainment-based FSWs may work at an establishment serving food or drinks, they also use this venue to find clients. Despite government restrictions, a few entertainment-based FSWs will spend time at an establishment waiting to meet clients; others will go to an entertainment venue only after receiving a call from a venue owner or employee that male patrons are requesting female companionship. Entertainment venues are used in TLS since they are easily identifiable and enumerated and the FSWs who work in them can be identified and approached for an interview.

Semi-visible FSWs Brothel, guesthouse and hotel FSWs are classified as semi-visible because they are not always easily identifiable. Most FSWs who reach their clients in hotels or guesthouses do so through the hotel concierge or an intermediary who calls them on behalf of a male guest. Guesthouse based FSWs are often paid less and spend less time with a client than a hotel based FSW. FSWs do not often spend time in or around hotels and guesthouses soliciting client. Brothels are extremely hidden in Vietnam, and the government does not recognize their existence. For the reasons cited above, these types of FSWs in Vietnam would not be appropriate for TLS. However, not enough is known about these types of FSWs in Vietnam to confidently categorize them as never being visible and therefore inaccessible with TLS. In many countries where brothels are more visible (i.e., parts of India, Cambodia, Nepal, etc.) FSWs are easily enumerated and sampled using TLS (FHI, 2000).

Non-visible The most hidden types of FSWs in Vietnam are those that reach their clients by telephone or through the Internet, an agent or a gatekeeper. It would be impossible for TLS to capture these types of FSWs unless they had access to a FSWs' telephone numbers, Internet addresses or agents. Then again, using lists of phone numbers, Internet addresses or contacts with agents in order to reach FSWs would not constitute TLS, but rather would involve other types of non-probability sampling techniques (e.g., snowball sampling, targeted sampling, etc.). For these reasons, it is accurate to classify these types of FSWs as non-visible.
} 
activity. The protocol for this study was reviewed and approved by the LIFE-GAP Office of the Vietnam Ministry of Health.

\section{FINDINGS}

A total of 628 individuals, including seeds, were interviewed in fixed interview sites in HCMC $(n=413)$ and HP $(n=215)$. The majority of the FSWs in the HCMC $(54.6 \%)$ and HP $(51.3 \%)$ sample were under the age of 29 years old. More than $50 \%$ of FSWs in HCMC and HP had no occupation other than selling sex.

During the follow-up visits to collect recruitment incentives, most participants reported giving out all of their coupons. According to the 174 recruiting participants in HCMC and 90 in HP, each of whom received three coupons, a total of $75(15.3 \%)$ individuals in HCMC and $24(10 \%)$ in HP refused to accept a coupon; 147 recruiters in HCMC and 83 in HP reported that they were successful in distributing their coupons and that no one to whom they tried to give a coupon rejected one; so refusals were clustered among a handful of recruiters, 27 in HCMC and 7 in HP. According to recruiters, most refusers did so because of "fear of identification," (57\% in HCMC; $75 \%$ in HP), others "felt uncomfortable" $(27 \%$ in HCMC; $8.3 \%$ in HP), still others thought they were "too busy" (10.7\% in HCMC), others thought the "incentive not worth the time" (17\% HP) and a small number of FSWs in HCMC responded that they were "not interested."

Overall, the recruited FSWs were diverse with respect to where they solicited their clients. Figure 1 depicts the diversity of sex work types among the links within a single recruitment chain of FSWs in HCMC ( $n=59$ recruits). In this example, the seed recruited three FSWs who were not street-based: two based in guesthouses and one based in karaoke." The selected karaoke-based FSW recruited two other karaoke-based and one guesthouse-based FSW. Near the outer waves of this recruitment chain, there are some more hidden types of FSWs such as agent-based and telephone-based FSWs.

The distribution by visibility was visible (HCMC, $n=311$; HP, $n=161$ ), semivisible (HCMC, $n=65$; HP, $n=43$ ) and non-visible (HCMC, $n=37$; HP, $n=12$ ). Figure 2 shows that the largest adjusted proportions of FSWs in both HCMC and HP reached by RDS were visible [HCMC, 73.7\%; 95\% ci $(67.0 \%, 79.5 \%)$ and HP, $75.9 \%$; ci $(67.1 \%, 85.3 \%)]$. RDS reached a small proportion of non-visible [HCMC, 9.3\%; ci $(6.0 \%, 13.1 \%)$ and HP, 9.7\%; ci $(1.3 \%, 18.2 \%)]$ and semivisible FSWs [HCMC, $16.9 \%$; ci $(11.5 \%, 23.4 \%)$ and $\mathrm{HP}, 14.2 \%$; $\operatorname{ci}(9.1 \%$, $21.2 \%)$ ], who theoretically could have been missed by TLS.

\section{HIV Risk Factors}

There were minor differences among FSW visibility groups with regard to HIV status and related risk behaviors (see Table 1).

HIV positive test status did not differ by visibility group in HCMC or HP. In HCMC and HP, a higher proportion of visible FSWs tested positive for HIV (14.5 and $35.2 \%$, respectively) than semi-visible $(13.8 \%, 30.2 \%)$ and non-visible (13.5 and

\footnotetext{
"Karaoke is the label used to represent all entertainment based FSWs, including those who work in bars and restaurants.
} 


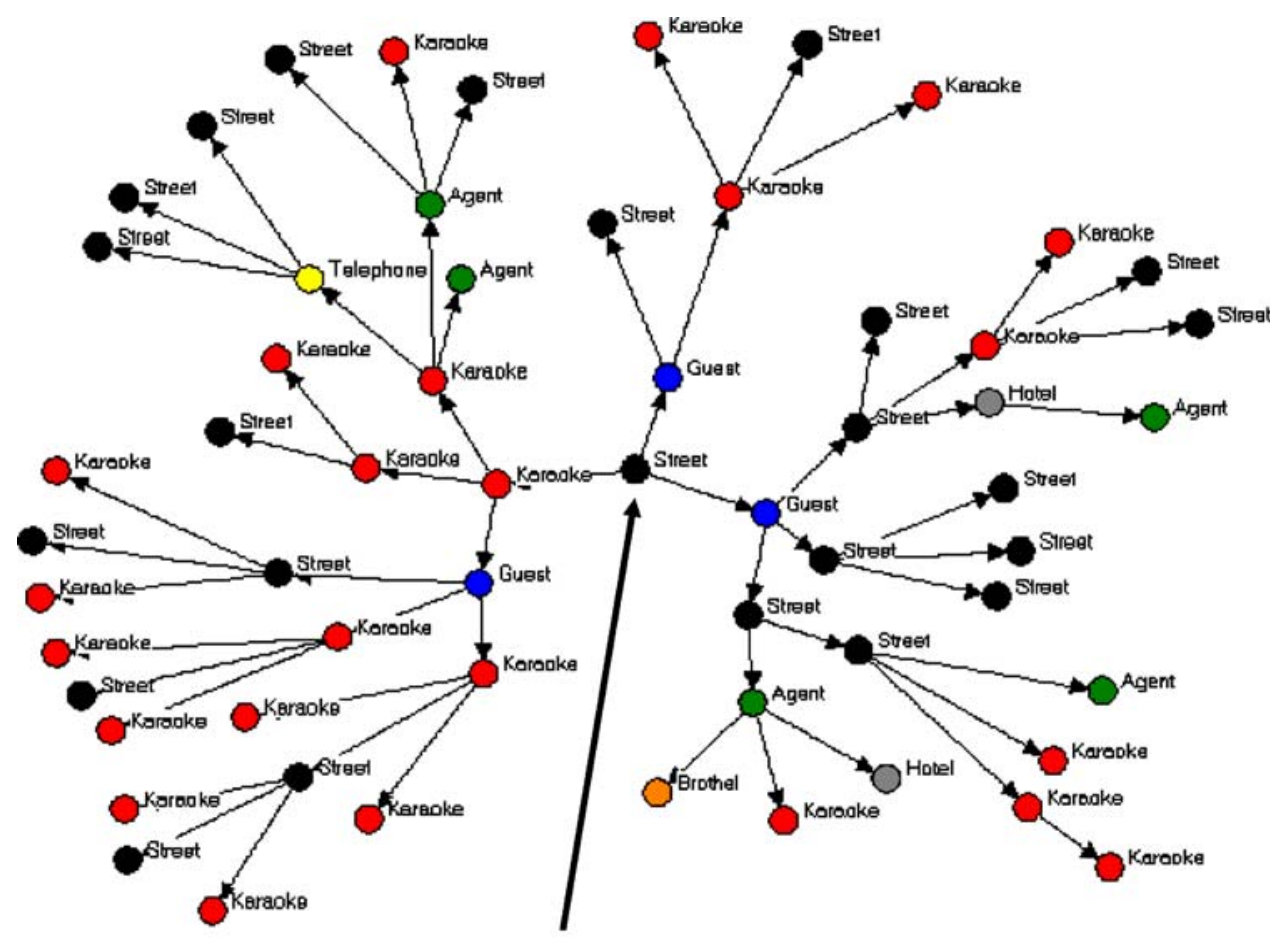

Seed

FIGURE 1. RDS recruitment network for one seed for type of female sex worker in HCMC, Vietnam.

$30.0 \%)$ FSWs. The only significant difference among the groups for any variable was in HP, for "no past month condom use with a partner" ( $p$ value $=0.009)$.

Visibility groups in both HCMC and HP did not differ with respect to reported use of non-injection illicit drugs ever and injected illicit drugs ever or reporting a sexually transmitted infection (STI) during the previous year.

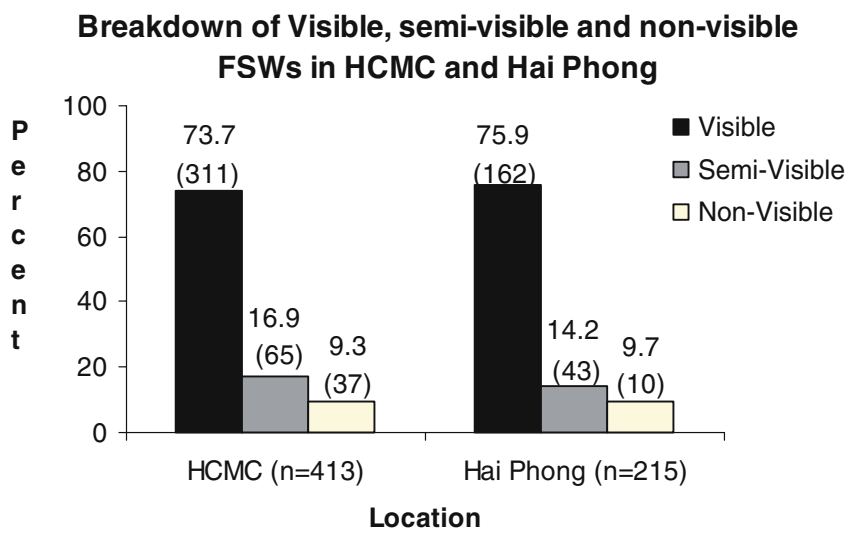

FIGURE 2. Distribution of visible, semi-visible and non-visible FSWs in HCMC and Hai Phong, Vietnam; adjusted proportions (number). Visible FSWs are assessed to be the most visible of the FSWs in Vietnam; semi-visible FSWs are assessed as not always being easily identifiable; and nonvisible FSWs are assessed as the most hidden types of FSWs in Vietnam. 


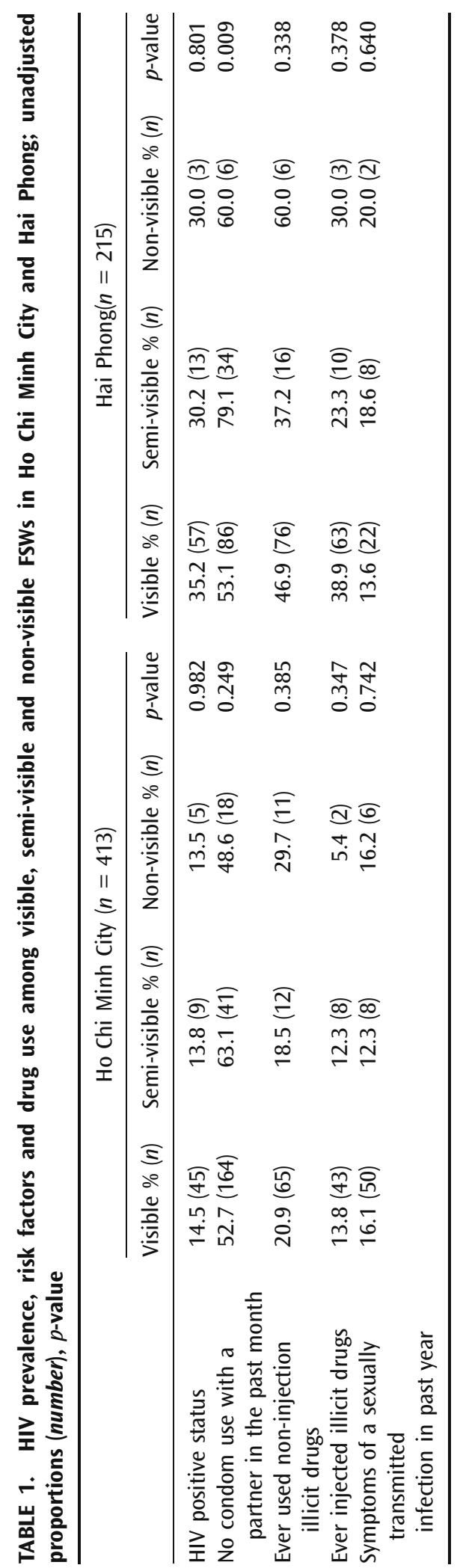




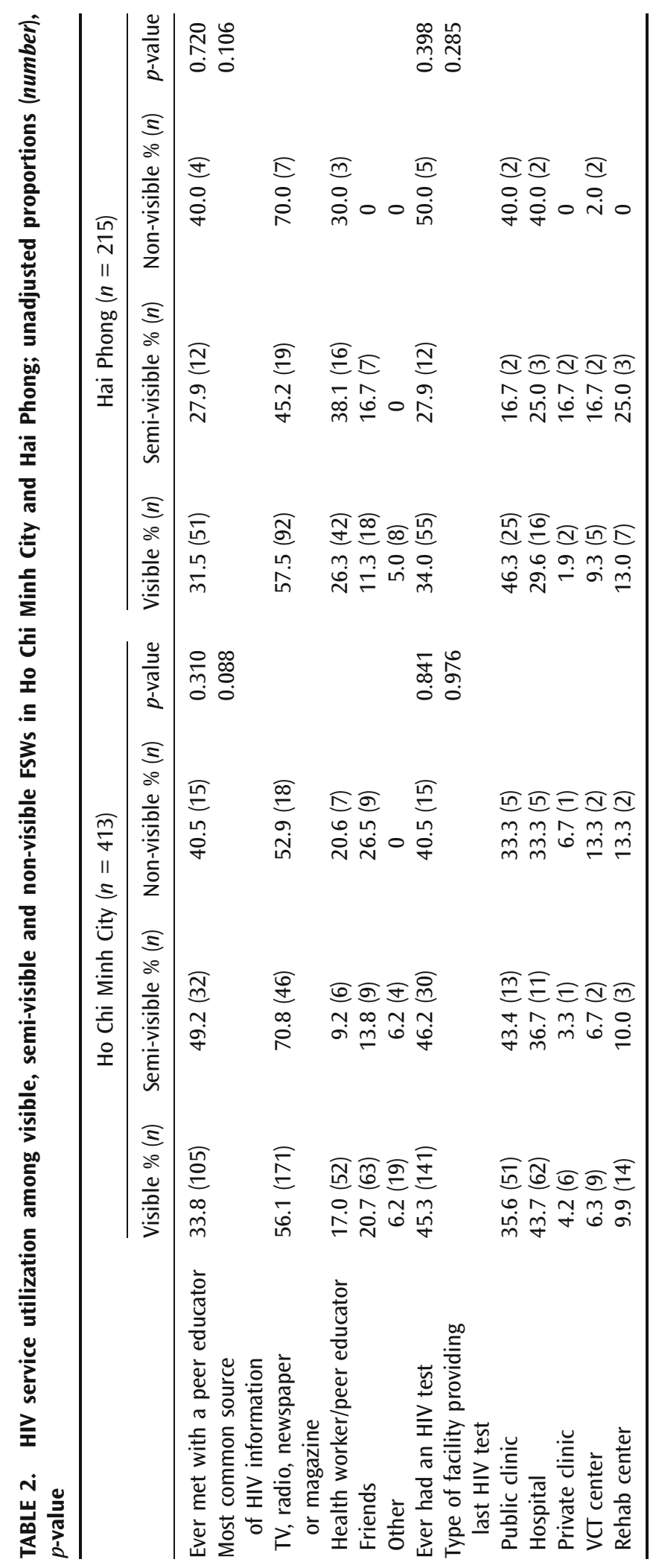




\section{Service Utilization}

Contact with a peer educator (PE), source of HIV information and HIV testing did not vary significantly by visibility group in either city. Among all three visibility groups in both cities, fewer than $50 \%$ had ever made contact with a PE. More than $50 \%$ of FSWs in both cities (with the exception of semi-visible FSWs in HP) reported receiving their HIV information from television, radio, newspapers or magazines. More HCMC FSWs reported receiving HIV information from friends than from health workers and PEs; this pattern was reversed for HP FSWs (Table 2).

Fifty percent or fewer FSWs in each visibility group reported ever having an HIV test. Among those HCMC and HP FSWs who ever had an HIV test, the majority received their test at either a public clinic (district or community public health center or STI clinic) or hospital, with the exception of HP semi-visible FSWs who reported receiving their HIV test from either a hospital or a drug user/sex worker rehabilitation center. Twice as many non-visible $(13.3 \%)$, in comparison to visible and semi-visible $(6.3 \%$ each) FSWs in HCMC, reported receiving their HIV test at a VCT center.

\section{DISCUSSION}

The analysis of RDS coverage demonstrates that this method may provide modestly better estimates for FSW populations than TLS because it reaches individuals ostensibly inaccessible by TLS. The level of risk and HIV prevalence in the less visible groups of FSWs was similar to the semi-visible and visible FSWs. This would indicate that less visible FSWs merit reasonable efforts to assure that these groups are targeted within all interventions for FSWs. The differences might have differed if two groups emerged rather than three. However, the semi-visible group could not be clearly dichotomized.

The three visibility groups in HCMC and HP did not differ significantly, which may contradict previous reports that higher paid, indirect FSWs, in general, have less HIV risk than direct FSWs. ${ }^{5,11,33}$ High proportions of respondents among all visibility groups in HCMC and HP reported inconsistent condom use with a sexual partner in the past month, possibly indicating that all three groups of FSWs are not being appropriately educated about using condoms or that they cannot easily access them. Other possible barriers to increased condom use might include poor negotiating skills with their clients or a false perception that a well-dressed client with an occupation may not be HIV-infected. Understanding the perceptions, negotiation skills and the reasons why some FSWs use condoms and others do not is essential to addressing the low proportion of condom use among FSWs in these cities.

The high proportion of non-visible FSWs in HCMC and HP who ever met with a $\mathrm{PE}$ indicates that the more hidden FSWs may be receiving more exposure to HIV information than previously believed. HCMC and HP FSWs reported similar sources for HIV information, indicating that PEs may be equally disseminated among the visibility groups. Furthermore the high proportion of non-visible FSWs who ever had an HIV test and their access to HIV testing at public clinics, hospitals and VCT centers indicate that this group has some awareness of and interest in their risk. It would be useful to gather qualitative data to determine how they obtained information and services.

Another key finding was the nature of social networks formed by FSWs in Vietnam. Networks were dense and diverse enough to provide a large sample in a short time that crossed the various types, direct and indirect, of FSWs working in the 
respective cities. These network properties are essential to the successful use of RDS. RDS had an added advantage over TLS by providing some measure of selection bias by questioning respondents on the characteristics of peers who demurred. TLS cannot determine reasons for not using a venue to solicit clients, only for respondents' refusal to speak with interviewers.

There were limitations to our study. Categories of visibility were based on the authors' perceptions of the sex work industry in the respective cities. We did not conduct extensive fieldwork so there was limited knowledge about the types of FSWs in Vietnam. There was too little formal research about characteristics of hotel and guesthouse-based FSWs to confidently dichotomize them as visible or non-visible. With a better understanding of these types of FSWs, reclassification and reanalysis of the data could better demonstrate the capacity of RDS to reach hidden FSWs. Furthermore, the question "Where do you usually reach your clients?" does not signify where a FSW always reaches her clients. The findings here may have nondifferentially misclassified some respondents.

Currently RDS data analysis is limited to estimates of proportions. Stratified analysis of the differences in risk factors among visibility groups might yield more information when RDSAT is updated.

The best scenario for testing whether RDS captures more hidden elements of the FSW population would be to conduct nearly contemporaneous surveys using TLS and RDS in the same population. Using RDS data to simulate TLS for comparisons of the two sampling methods is subject to any biases unique to RDS that might not be found in TLS.

Although non-visible FSWs make up a small proportion of the FSW sample in HCMC, their high HIV prevalence and associated risk factors is alarming, especially when past efforts to sample FSWs, using TLS and convenience sampling, and sentinel surveillance in STI clinics, are likely to have missed these groups. Missing the most inaccessible portions of the FSW population could result in a biased understanding of HIV sero-prevalence and risk factors and, consequently, could lead to inefficient planning for HIV prevention and intervention programs for the entire FSW population. Recurrent round-ups of FSWs drive FSWs off the street, making them increasingly difficult to find in public venues. If current prevention programs are targeting and responding to those who are most likely to be assessed (more visible FSWs) then it is possible that an important segment of the FSW population at risk for HIV is not receiving optimal HIV information and services. However, this study found that segments were accessing services at about the same low rates, indicating a need to increase service utilization among all groups of FSWs.

Based on these findings, we recommend using RDS for future surveys of FSWs in Vietnam to prevent the omission of the less accessible members of this population.

\section{ACKNOWLEDGEMENTS}

This study was funded by the US government through a Cooperative Agreement between the Centers for Disease Control and Prevention-Global AIDS Program and the Vietnamese Ministry of Health. The authors wish to thank the Ho Chi Minh City and Hai Phong participants for their contributions. We also thank the staff of the local public health centers and other establishments used as interview sites and the peer outreach workers who helped select our initial sex worker participants for this study. 
Disclaimer: The findings and conclusions in this paper are those of the authors and do not necessarily represent those of the Centers for Disease Control and Prevention.

\section{REFERENCES}

1. Vietnam Technical Working Group on HIV Estimates and Projections (VTWG). Briefing Paper on Vietnam Estimates and Projections, 2003. Vietnam Technical Working Group on HIV Estimates and Projections. Hanoi, Vietnam; 2004.

2. World Health Organization. The AIDS Epidemic in Vietnam has not Reached its Peak. Press Release. Hanoi, Vietnam; 2002.

3. Nguyen DT, Nguyen AT, Hoang TV, et al. HIV/AIDS Behavioral Surveillance Survey, Vietnam, 2000. National AIDS Standing Bureau; Family Health International. Hanoi, Vietnam; 2001.

4. Vietnam News. Proposed Karaoke Ban Faces Wide Protest. Hanoi, Vietnam; 2004, February 24. Available at: http://vietnamnews/vnanet.vn.(Personal communication, David Trees, World Vision, Hanoi, 2004)

5. Trees, D. HIV and STI Prevention with Sex Workers in Vietnam: The Literature. UNFPA/ World Vision. Hanoi, Vietnam; 2003.

6. Nhu TDH, De Bruin L, Starink, M. Urban Migration and Vulnerability Amongst Sex Workers in HCMC. Abstract presented at: The XIV International AIDS Conference. Barcelona, Spain; 2002. Available at: www.unaids.org.vn/resource/topic/abstract/6icaap/ 1720.RTF.

7. Starink M, De Bruin L. Mobility and Vulnerability: An explorative study of female sex workers in Ho Chi Minh City, Vietnam. Mobility Research and Support Centre-Coordination of Action Research on AIDS and Migration. Ho Chi Minh City, Vietnam; 2001. Available at: http://www.unaids.org.vn/resource/topicindex/sexwork.htm.

8. UNAIDS. Population Mobility and AIDS. UNAIDS Technical Update. Geneva, Switzerland; 2001.

9. Tep M, Ek S. Vietnamese Sex Workers in Cambodia. CARAM: Research in Sex Work 3. Phnom Pen, Cambodia; 2000. Available at: http://www.nswp.org/r4sw/index.html and http://www.nswp.org/pdf/R4SW-03.PDF.

10. Rekart ML. Sex in the city: sexual behavior, societal change and STDs in Saigon. Sex Transm Infect. 2001;78(Suppl 1):i47-i54.

11. Nguyen AT, Nguyen TH, Pham KC, et al. Intravenous drug use among street based sex workers. Sex Transm Dis. 2004;31(1):15-19.

12. Doussantousse S, Tooke L. Women involved in prostitution in Vietnam-a Hanoi snapshot. Hanoi, Vietnam. 2002. Available at: www.unaids.org.vn/resource/topic/sexwork/ CSW prostitution.doc.

13. Nguyen MT, Vu TH, Blanc ME. Sexual behavior related to HIV/AIDS: commercial sex and condom use in Hanoi, Vietnam. Asia Pac Popul J. 2002;17(3):33-38.

14. Nguyen TT, Lindan C, Nguyen XH, Barclay J, Khiem HB. Sexual risk behavior of women in entertainment services in Vietnam (Vung Tau Province). AIDS Behav. 2000;4:93-101.

15. Nguyen TT, Vo TN, Nguyen VT, Truong XL, Ha BK. HIV infection and risk factors among female sex workers in southern Vietnam. AIDS. 1998;12:425-432.

16. Franklin B. The Risk of AIDS in Vietnam: An Audience Analysis of Risk Factors for HIV/AIDS Amongst Men and CSWs in Hanoi and HCM City. CARE International in Vietnam Monograph Series No. 1. Hanoi, Vietnam; 1993.

17. Nguyen AT, Bui DT, Nguyen TH, et al. Baseline survey report in Lai Chau, Quang Tri, Dong Thap, An Giang and Kien Giang. Community Action for Preventing HIV/AIDS Project. CARE International in Vietnam Monograph Series No. 1. Hanoi, Vietnam; 2003.

18. Family Health International. HIV/AIDS Behavioral Surveillance Survey: Vietnam, Round 1. FHI/IMPACT and USAID. Arlington, Virginia; 2001. 
19. Heckathorn DD, Jeffri J. Jazz Networks: Using Respondent Driven Sampling to Study Stratification in Two Jazz Musician Communities. Paper presented at the American Sociological Association. Atlanta, Georgia; 2003, August 16-19.

20. Heckathorn DD. Respondent driven sampling II: deriving valid population estimates from chain-referral samples of hidden populations. Soc Probl. 2002;49(1):11-34.

21. Heckathorn DD, Jeffri J. Finding the beat: using respondent-driven sampling to study jazz musicians. Poetics. 2001;28:307-329.

22. Heckathorn DD. Respondent driven sampling: a new approach to the study of hidden populations. Soc Probl. 1997;44(2):174-199

23. Semaan S, Lauby J, Liebman J. Street and network sampling in evaluation studies of HIV risk-reduction interventions. AIDS Rev. 2002;4:213-223.

24. Muhib FB, Lin LS, Stueve A, et al. A venue-based method for sampling hard-to-reach populations. Public Health Rep. 2001;116(Suppl 1):216-222.

25. Family Health International. Evaluating Programs for HIV/AIDS Prevention and Care in Developing Countries: A Handbook for Program Managers Decision Makers. FHI. Arlington, Virginia; 2001.

26. Stueve A, Odonnell L, Duran R, Doval A, Blome J. Methodological issues in time-space sampling in minority communities: results with Latino young men who have sex with men. Am J Public Health. 2001;91:922-926.

27. Abdul-Quader AS, Heckathorn DD, McKnight C, et al. Effectiveness of respondent driven sampling for recruiting drug users in New York City: findings from a pilot study. J Urban Health. 2006;83:459-476.

28. Ramirez-Valles J, Heckathorn DD, Vasquez R, Diaz RM, Campbell RT. Respondent driven sampling among gay men: lessons from a two-city field study with Latino gay men. AIDS Behav. 2005; DOI 10.1007/s10461-005-9012-3.

29. Heckathorn DD, Semaan S, Broadhead RS, Hughes J. Extensions of respondent driven sampling: a new approach to the study of injection drug users aged 18-25. AIDS Behav. 2002;6(1):55-68.

30. Heckathorn, DD. RDSAT, version 5.0. Available at: http://www.respondentdrivensampling.org. Cornell University, Ithaca, New York; 2005.

31. Salganik MJ and Heckathorn DD. Sampling and estimation in hidden populations using respondent-driven sampling. Sociol Method. 2004;34:193-239.

32. Erickson, BH. Some problems of inference from chain data. Sociol Method. 1979;10:276-302.

33. Elmer L. HIV/AIDS intervention data on commercial sex workers in Vietnam: A review of recent research findings. National AIDS Standing Bureau. Hanoi, Vietnam; 2001. Available at: http://www.unaids.org.vn/resource/topicindex/sexwork.htm. 